export. In combination with the short-cut to decay, this would prevent production of an incorrect protein.

Prokaryotes have a great deal to teach us about mRNA stability. The structure at the $5^{\prime}$ ends of prokaryotic mRNAs is clearly important for message stability and its regulation by specific endonucleases, although the precise mechanisms remain unknown. Some prokaryotic ribonucleases seem to work in complexes with other proteins - for example RNaseE is found with polynucleotide phosphorylase and with the chaperone protein GroEL. Work with human cells has uncovered activities that may be comparable to bacterial RNases.

Once a correctly processed and (at least temporarily) stable message is available, it must still be selected for translation. Detailed studies of eukaryotic translational initiation factors (eIFs) have allowed analysis of the RNA-protein interactions that guide the ribosome to the start of a reading frame. New RNA-binding proteins that act in translational control have been discovered. One of these, the p50 component of messenger ribonucleoprotein, may be a general repressor of translation, as well as a transcription factor; and another, previously identified as the La autoantigen recognized by autoantibodies from systemic lupus patients, might be involved in translational initiation at internal ribosome entry sites. Although the translation of most mRNAs depends on the mRNA cap and ribosome scanning from the $5^{\prime}$ end of the RNA, internal initiation is the mechanism of choice for picornaviruses. The RNA sequences and protein factors responsible for internal ribosome entry in picornaviruses are now being identified. They may also be involved in translation of some mammalian mRNAs

In prokaryotes, transcription and translation occur in the same compartment and are tightly coupled. Decoupling the two processes by using a very fast RNA polymerase leads to destabilization of mRNAs, suggesting that coupling of the two processes prevents such decay. In eukaryotes, where transcription and translation take place in different cell compartments, it is still unclear to what extent some form of coupling over the nuclear membrane is possible.

Eukaryotic translation can be downregulated by phosphorylation of factor eIF- $2 \alpha$, as occurs in response to stress, or of the elongation factor eEF-2, phosphorylation of which seems to be involved in insulin regulation of gene expression. But translation is enhanced during Xenopus oocyte maturation by protein interactions with specific signals in the $3^{\prime}$ UTRs of certain mRNAs; these signals mediate selective polyadenylation and thus translation of the messages. Moreover RNAprotein interactions that inhibit transla-

OPTICS

\title{
Reflections on a summer's night
}

ALISTAIR B. Fraser of the Pennsylvania State Uni. versity evidently has a fatigue-defying appetite for meteorological curiosities. The glistening tree shown here is a fine example of a phenomenon that he first spotted in the small hours of a summer's morning, whilst driving along a forested road in British Columbia. Fraser describes his experience in a article to appear shortly in a special issue of Applied Optics (vol. 33, 20 July 1994): in his words, he noticed that in the car's headlights "the trees of the normally Stygian forest began to glow as

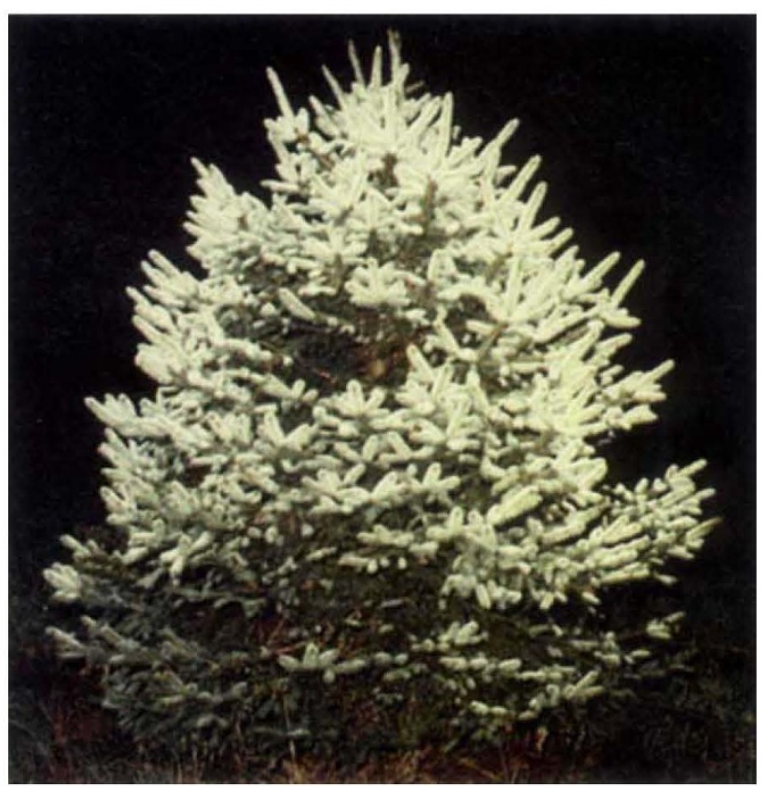
if snow-covered", a startling observation on a warm August night. Evidently some form of retroflection from dew-covered leaves, the effect seemed oddly speciesspecific, even on a first inspection. Later nocturnal expeditions with a powerful flashlight (a proceeding that aroused dark suspicions in at least one local gamekeeper) showed that it favoured only certain types of conifer and a few shrubs such as the yew and rhododendron. The explanation lies in the contact angle of droplets on the leaves: as this rises above 90 degrees or so, the proportion of light from the car's headlamps that is reflected back towards the occupant increases, and for angles above about 140 degrees, the retroreflection becomes spectacular. Blue spruces show the glow particularly well, because the tangled rod-like morphology of the epicuticular wax responsible for their blue bloom also increases the contact angle. Fraser dubs the glow sylvanshine, in deference to the heiligenschein "familiar to those who wander grassy fields in the early morning". But whereas the sylvanshine on a tree is visible to anyone in the car or holding a torch, heiligenschein, the result of sunlight focused by dewdrops held above a leaf's surface by fine hairs, appears to each observer as a halo around the head of just his own shadow on the grass. In his Memoirs of 1562, Benvenuto Cellini, no expert on the laws of optics, interpreted this instead as a sign of divine grace towards himself; and, says Fraser wryly, evidently none of those to whom Cellini vouchsafed his secret dared mention the halo about his own head.

Lindsay Matthews

tion can be used to characterize and clone new RNA-binding proteins.

Our picture of prokaryotic translation is also changing, as the third release factor for translational termination (RF3), which stimulates the other two, has now been found. Other prokaryotic insights include translational 'jumping', by which the ribosome bypasses a 55-base segment of mRNA, the incorporation of selenocysteine into proteins, which also occurs in eukaryotic cells, and the translational auto-regulation of threonyl-transfer RNA synthetases by 'molecular mimicry'; here, both the protein and its cognate tRNA bind to a specific codon in the synthetase mRNA leader. Translation of some prokaryotic RNAs is inhibited by ribosome trapping, for which specific RNA pseudoknot structures are required.

In all of these processes, RNA structure is crucial. As studies of RNA crosslinking and ribosomal protein protection improve our three-dimensional view of ribosomal RNA structure, an increasing number of correlations between structure and function are emerging. The importance of RNA structure in controlling processes as diverse as translational frameshifting, ribosome binding and antisense-mediated control of plasmid replication emphasizes the versatility of RNA.

RNA was once regarded as a mere passive product of the real controller of gene expression, transcription. But it is now clear that it is central to the actions of the spliceosome and ribosome, as well as all the other processes of post-transcriptional regulation. We can expect to see a great deal of action as scrutiny of RNA versatility becomes more intense. $\square$

Kimberly Carr is an assistant editor at Nature. 